REGARDS

SUR LEECONOMIE ALLEMAND

BULLETIN ECONOMIQUE DU CIRAC
Regards sur l'économie allemande

Bulletin économique du CIRAC

95 | 2010

Varia

\title{
Confiance dans la sortie de crise
}

Isabelle Bourgeois

\section{OpenEdition}

Journals

Édition électronique

URL : https://journals.openedition.org/rea/4015

DOI : 10.4000/rea.4015

ISBN : 978-2-8218-0886-7

ISSN : 1965-0787

Éditeur

CIRAC

Édition imprimée

Date de publication : 1 mars 2010

Pagination : 3-4

ISSN : 1156-8992

Référence électronique

Isabelle Bourgeois, "Confiance dans la sortie de crise », Regards sur l'économie allemande [En ligne] 95 | mars 2010, mis en ligne le 08 mars 2010, consulté le 21 septembre 2021. URL : http:// journals.openedition.org/rea/4015; DOI : https://doi.org/10.4000/rea.4015 


\section{Confiance dans la sortie de crise}

L'Allemagne semble avoir rompu avec la récession. Après une chute historique de $-5 \%$ du PIB en 2009 (chiffre confirmé le 24 février par Destatis), l'année 2010 s'annonce bien meilleure. Reste à savoir de combien croîtra le PIB : de $+1,4 \%$ comme l'affirme le gouvernement fédéral dans son rapport annuel, ou de $+2,3 \%$ comme le pense la Fédération des $\mathrm{CCl}$ allemandes DIHK à l'issue de son enquête de janvier ? Difficile à dire, tant l'ampleur du redémarrage des exportations dépend à la fois de la durée de l'impact des mesures conjoncturelles adoptées dans les pays industrialisés et de la vigueur de la demande des économies au sein de la zone Euro. Car « les forces endogènes de croissance ne sont pas encore assises sur un fondement assez large et solide », explique la Bundesbank dans son rapport de février.

Il est vrai que l'économie allemande, dont les principaux indicateurs montrent actuellement des signes de reprise parfois nets, part d'un niveau très bas. Au $4^{e}$ trimestre 2009 , le PIB a stagné en comparaison du trimestre précédent. La consommation intérieure s'était tassée $(-2,1 \%)$ sous l'effet d'une baisse des dépenses de consommation publiques $(-0,6 \%)$, de celles des ménages $(-1,0 \%)$ et, surtout, du recul des investissements $(-0,5 \%$ pour la construction, $-1,5 \%$ pour les équipements). Cette tendance a été compensée par un excédent commercial dont la contribution au PIB avait été de 2 points ; la reprise des exportations s'était poursuivie $\left(+3,0 \%\right.$ après $+3,4 \%$ au $3^{\mathrm{e}}$ trimestre), alors que les importations avaient à nouveau reculé $(-1,8 \%)$ après la hausse de $+5,2 \%$ qu'elles avaient enregistrée au trimestre précédent (la seule en 2009). Autrement dit, avec le regain de l'activité dans les pays asiatiques, puis aux USA, dans les pays de l'OPEC et de l'Europe centrale et orientale, le principal moteur de l'économie allemande a redémarré à la mi-2009.

\begin{tabular}{|c|c|c|c|c|c|c|c|}
\hline \multicolumn{7}{|c|}{ Evolution trimestrielle de la croissance allemande en 2008 et $\mathbf{2 0 0 9}$} \\
I & II & III & IV & I & II & III & IV \\
& \\
$1,6 \%$ & $-0,6 \%$ & $-0,3 \%$ & $-2,4 \%$ & $-3,5 \%$ & $0,4 \%$ & $0,7 \%$ & $0,0 \%$ \\
$2,1 \%$ & $3,4 \%$ & $1,4 \%$ & $-1,7 \%$ & $-6,4 \%$ & $-7,0 \%$ & $-4,7 \%$ & $-1,7 \%$ \\
\hline
\end{tabular}

Source des données : Destatis, communiqué du 24-02-2010.

II n'en reste pas moins que, sur l'ensemble de l'année, les exportations ont chuté de $18,4 \%$ en valeur par rapport à 2008 (Destatis). Ce recul historique (très net pour les biens d'investissement et les biens intermédiaires) fait dire à plusieurs fédérations qu'il faudra plusieurs années pour qu'elles retrouvent leur niveau d'avant la crise - jusqu'en 2014, à en croire la fédération de l'industrie (BDI). Quant au fait que l'Allemagne a dû céder son titre de championne du monde à l'export à la Chine, « ce n'est pas un souci pour nous », déclare Anton Börner, président de la fédération du commerce extérieur (BGA), puisque « la croissance de l'économie chinoise va assurer la nôtre et garantir nos emplois pour de longues années encore » (déclaration du 09-02). Les acteurs industriels sont visiblement conscients du dynamisme retrouvé de la demande extérieure, comme le confirme l'indice ifo de février. Certes, celui-ci n'a pas grimpé pour la première fois depuis dix mois, mais c'est seulement parce que les entreprises se disent un peu moins satisfaites de l'état actuel de leurs activités ; elles sont nettement plus confiantes quant à leur évolution au cours des six prochains mois. De son côté, l'enquête réalisée en janvier par la DIHK auprès de ses membres révèle que les entreprises, tous secteurs confondus, ont retrouvé le moral: plus des trois quarts d'entre elles jugent leur situation satisfaisante ou bonne, et elles sont sereines quant à l'avenir (plus de la moitié n'envisage pas de changement, un gros quart s'attend à une amélioration). Leur confiance repose principalement sur les perspectives à l'export.

Si la production industrielle $(+1,0 \%$ au dernier trimestre) a perdu en dynamique à la fin de l'année $(-2,8 \%$ de novembre à décembre selon le rapport du gouvernement), cela est dû au contrecoup de la fin de la prime à la casse «qui a eu un large impact sur la conjoncture », précise la Bundesbank. Dans l'ensemble, même si le taux d'utilisation des capacités reste en deçà de son niveau d'avant la crise, il progresse, se

Les entreprises voient l'avenir avec confiance

Tendance à la reprise de l'investissement 
Stabilité de l'emploi...

... et de la consommation

Priorité absolue à la consolidation budgétaire... en 2011 seulement situant désormais à un peu plus de $75 \%$ de la moyenne antérieure (4 points de plus que son point le plus bas en avril dernier selon les sondages de l'institut ifo). Les entreprises semblent à nouveau enclines à investir pour renouveler ou moderniser leurs capacités, voire les étendre, principalement dans l'industrie chimique ou l'automobile ; les prestataires de services de R\&D sont à la pointe de cette tendance, ce qui est « un signal positif » pour l'innovation en Allemagne (DIHK).

Malgré le faible taux d'utilisation de leurs capacités, les entreprises évitent, dans la mesure du possible, de réduire leurs effectifs, préférant épuiser toutes les possibilités que leur offre un droit du travail aujourd'hui flexible et recourir au chômage partiel pour conserver leurs compétences. Selon l'Institut IAB, cela a permis de sauver jusqu'ici 1,2 million d'emplois, l'équivalent du recul de $3,2 \%$ de la durée de travail moyenne sur l'année (elle a atteint 1309 heures par salarié). Dans l'ensemble, la productivité horaire a baissé de $2,1 \%$. Mais elle devrait reprendre, seuls quelques groupes du Dax-30 recourant encore au chômage partiel. Au total, de février 2009 à février 2010, le nombre de chômeurs indemnisés n'a augmenté que de 91000 , passant à 3,643 millions (soit un taux de 8,7 \% selon l'Agence de Nuremberg).

"Le fort tassement de la production ne se répercute ainsi que très faiblement sur le marché de l'emploi... et n'a que très peu affecté l'évolution de la consommation », résume la Bundesbank. Le moral des ménages a été soutenu en outre par une hausse de $2,8 \%$ en moyenne des salaires mensuels en 2009 (+3,0 \% dans l'industrie) et un taux d'inflation de $0,4 \%$ seulement, essentiellement dû à l'énergie (Destatis). Certes, le revenu disponible des ménages n'a augmenté que de $0,4 \%$, tombant à son niveau des années 1990, mais le taux d'épargne est resté à $11,2 \%$ comme en 2008. «La crise n'a pas atteint les ménages, ... n'affectant que les revenus des entreprises et du patrimoine », conclut Klaus Zimmermann, président de l'institut DIW (IZA Standpunkte, 22/2009). Et de fait, ces derniers ont chuté de $11 \%$ en 2009 (Destatis).

Les mesures de soutien de l'économie (et de la consommation), qui n'ont pas toutes encore, loin s'en faut, déployé leurs effets, ont « laissé une profonde empreinte dans les budgets publics » (Bundesbank). Les dépenses publiques ont augmenté de $5 \%$ en 2009 ; le déficit a atteint 79,1 milliards $€$ en 2009 (dont la moitié imputable au Bund), passant à $3,3 \%$ du PIB. Le gouvernement fédéral s'est engagé à le ramener en-dessous des $3 \%$ d'ici 2013 en le réduisant de $0,5 \%$ du PIB par an à partir de 2011 afin de respecter à la fois de Pacte de Stabilité et de Croissance et les nouvelles dispositions de la Loi fondamentale (surnommées « frein à l'endettement »). " Mais au regard des déficits élevés comme d'une dette qui continuera à augmenter en 2013, des efforts plus importants seraient souhaitables », rappelle la Bundesbank ; car, "il revient à l'Allemagne un rôle primordial pour faire prévaloir la nature contraignante des engagements contractuels de politique budgétaire dans l'UEM ». Cela « relève des travaux d'Hercule », avouait Wolfgang Schäuble, ministre fédéral des Finances, en ouverture des débats budgétaires au Bundestag (FAZ.NET, 19-02) où il annonçait des mesures drastiques, mais sans plus de précisions ; les axes concrets de la politique de rigueur ne seront connus qu'en juin. L'opinion est préparée.

QUE SEUL UN EFFORT COLLECTIF permettra à l'Allemagne de renouer durablement avec la croissance fait consensus outre-Rhin. En janvier, $58 \%$ des contribuables se disaient opposés aux projets de réduction des impôts de la coalition fédérale, et même $69 \%$ des sondés aux revenus mensuels supérieurs à $3000 €$ (Deutschlandtrend, ARD/Welt Online). Quant aux partenaires sociaux, ils viennent de démontrer dans la métallurgie le sens des responsabilités qui est le leur face à des enjeux primordiaux: en Rhénanie du Nord-Westphalie, IG Metall et son homologue patronal Gesamtmetall viennent de conclure un accord-pilote par lequel ils renoncent cette année à une hausse salariale en échange d'une garantie de l'emploi. Quant aux experts, ils plaident pour poursuivre les réformes structurelles et mieux investir dans les infrastructures comme les qualifications. Ils entendent par là une politique développant la flexibilité, condition première de la capacité d'adaptation à un environnement en perpétuelle évolution, autrement dit : un soutien résolu à la qualification d'individus aptes à gérer le changement. "Le creux de la vague est dépassé, mais remonter la pente sera long et pénible », c'est en ces termes que Rainer Brüderle, ministre fédéral de l'Economie, résumait les perspectives à moyen terme de l'économie allemande (FAZ.NET, 27-01). 